\title{
Spiritualität, Migration und Psychiatrie
}

\section{Ein heisses Thema für die Psychotherapie?}

\author{
Michele Mattia
}

Psychotherapie-Wissenschaft 9 (1) 69-75 2019

www.psychotherapie-wissenschaft.info

CC BY-NC-ND

https://doi.org/10.30820/1664-9583-2019-1-69

\begin{abstract}
Zusammenfassung: Die psychiatrische und psychologische Tätigkeit wird seit Jahrhunderten ausgeübt. Dennoch haben religiöse und spirituelle Fragen weiterhin Auswirkungen auf die Perspektiven der Patienten, insbesondere in Bezug auf ihre Gesundheit und dem Umgang mit den psychischen Störungen, von denen sie betroffen sind. Religiöse und spirituelle Überzeugungen sind weit verbreitet, mehr als man denken könnte, unter all denen, die an einer affektiven, psychotischen oder Angststörung leiden. Selten sind sich Psychotherapeuten und Psychiater der Bedeutung bewusst, die Religion im psychischen Konstrukt des Subjekts einnehmen kann. Es fällt ihnen schwer, Werte wie Spiritualität und Religion innerhalb der Strukturierung des psychischen Apparats der Menschen zu verstehen und nachzuvollziehen, wie weit diese Werte die Beziehungen zur Familie, zur Gesellschaft, zur Arbeit oder zur Konstruktion der eigenen persönlichen Identität unterstützen oder beeinträchtigen können. Dieser Artikel stellt einzelne klinische Fälle vor, um den Zusammenhang zwischen Religion, Spiritualität und psychiatrischen Störungen zu diskutieren. Insbesondere werden die fördernden, induzierenden oder schützenden Auswirkungen der verschiedenen religiösen Praktiken auf die jeweiligen Pathologien hervorgehoben. Wir möchten besonders das Verhältnis zwischen den drei monotheistischen Weltreligionen (Christentum, Islam und Judentum) und den anderen drei grossen Religionen Buddhismus und Hinduismus sowie der Evangelischen Kirche untersuchen. Es kommen sechs klinische Fälle zur Darstellung. In einem Fall geht es um Depressionen und Angstzustände und die schützende Wirkung einiger hinduistischer Praktiken. Zwei weitere Fälle fokussieren auf depressive Symptome und die Schutzwirkung der katholischen Religion. Ein weiterer Fall behandelt den religiösen Glaubenswechsel am Beispiel eines jungen Schweizer Philosophiestudenten. Er war Katholik und wechselte zum Islam. Diese Veränderung führte zunächst zu einem signifikanten Rückgang der psychiatrischen Störungen (er litt an einer besonders schweren Neurose), entwickelte sich aber später in einen aggressiven und gewalttätigen Zustand. Sein Geist fand keine Ruhe und entwickelte ein starkes Risiko der Radikalisierung. In einem weiteren Fall wird die lindernde Wirkung der meditativen Praktiken des Buddhismus verdeutlicht. Ein junger Schweizer, der seit vielen Jahren an verschiedenen Arten von Schmerz litt, entwickelte nach der Begegnung mit der buddhistischen Religion die Fähigkeit, seine Schmerzen zu bewältigen und den Konsum von Analgetika und Antidepressiva deutlich zu reduzieren. Im letzten Fall wird der schützende und entspannende Einfluss der evangelischen Kirche bei einer Frau mit Störung durch Panikattacken und hysterischem Bolus untersucht. Wir werden auch die Vorurteile analysieren, die den Psychotherapeuten in seiner klinischen Praxis blockieren können, und die Muster, mit denen er sich auseinandersetzen muss, um sich aus seinen inneren Blockaden zu befreien.
\end{abstract}

Schlüsselwörter: Kulturpsychiatrie, Spiritualität, Religion, Überzeugungen, Bewältigungsstrategie (Coping), Vorurteile, Psychotherapie

\section{Einführung}

«Gott, Götter, göttliche Macht oder Geister existieren in allen Kulturen und auf der ganzen Welt.» Koenig et al. (2001)

Religionen und Überzeugungen haben einen erheblichen Einfluss auf die Menschen und ihre psychische und somatische Gesundheit und den Umgang mit den Störungen, mit denen sie konfrontiert sind. Religiöse Überzeugungen sind laut den meisten Untersuchungen besonders bei Personen vorhanden, die unter einer emotionalen Störung oder einer psychischen/seelischen Krankheit leiden.

Ärzte sind sich der Bedeutung religiöser Überzeugungen selten bewusst und deren Verständnis würde der Behandlung mehr therapeutische Kraft verleihen (Koenig et al., 2005). Die wissenschaftlichen und theologischen
Gemeinschaften sind sich darüber uneins, wie man die Begriffe Religion und Spiritualität definieren soll. Der Begriff Religion bezeichnet spezifische, soziale und mit der Lehre verbundene Verhaltensweisen und Merkmale von Gruppen, die den verschiedenen religiösen Bereichen angehören. Insbesondere beinhaltet der Begriff Religion den Glauben an eine übernatürliche Macht oder an ein transzendentes Wesen, an eine Wahrheit oder höchste Wirklichkeit, und dieser Glauben kommt in Verhaltensweisen und Ritualen zum Ausdruck. Religion ist ein organisiertes System Überzeugungen, Praktiken und Ritualen, die sich am Heilige orientieren. 
Spiritualität ist ein Begriff, der die höchste Frage nach dem Sinn des Lebens betrifft und alles, was mit Transzendenz zu tun hat, beinhaltet. Er kann aus traditionellen religiösen Formen entstehen kann oder auch nicht (wird jedoch meist von diesen abgeleitet). Bei der Spiritualität geht es auch um persönliche Erfahrungen oder um die Erforschung des Transzendenten. Sie braucht nicht unbedingt mit einer bestimmten Religion zusammenzuhängen, sie geht über die blosse Zugehörigkeit zu einer Religion hinaus. Einige weisen darauf hin, dass die Definition von Spiritualität subjektiver und weniger messbar sei. Aus klinischer Sicht ist ein so weitgefasster und verbreiteter Begriff positiv, weil er es jedem ermöglicht, seine eigene Bedeutung für sich zu definieren und gleichzeitig die Subjektivität zu bewahren. Die Sprache der Spiritualität hilft, einen Dialog mit religiösen oder auch nicht-religiösen Menschen aufzunehmen (Larson et al., 1997).

\section{Die Rolle des Therapeuten}

Die Rolle des Arztes in diesen Situationen ist nicht offensichtlich. Psychotherapeuten gehen davon aus, dass sie vielfache Gründe haben, ihre Zurückhaltung gegenüber spirituellen und religiösen Themen in Therapien hervorzuheben.

In erster Linie haben Psychotherapeuten ihren eigenen religiösen Bezug - bzw. mangelnden religiösen Bezug -, der ihr Interesse an religiösen oder spirituellen Fragen beeinflussen kann. Im Allgemeinen sind Psychotherapeuten weniger an religiösen Aktivitäten beteiligt als Patienten, was zur Folge hat, dass sie weniger daran interessiert sind, diese Themen in der Therapie zu diskutieren (Neeleman \& King, 1993).

In zweiter Linie verfügen Psychotherapeuten über geringe Kenntnis, wie der religiöse und spirituelle Diskurs im klinischen Setting aufgebaut werden soll. Während des Hochschul- oder Postgraduiertenstudiums gibt es selten spezifische Ausbildungskurse zum Thema Religion oder Spiritualität. Man darf ausserdem nicht vergessen, dass es in der Vergangenheit immer wieder bedeutende Konflikte zwischen Psychiatrie, Psychologie und Religion gegeben hat.

Freud beschrieb die Religion als «obsessive Neurose der Menschheit», eine neurotische Verteidigung gegen die Wechselhaftigkeit der Existenz. Er war überzeugt, dass es den Menschen ohne religiösen Glauben besser gehen würde (Gay, 1987). Dieses nachteilige Bild wurde von Fachleuten der psychiatrischen Berufe oft hervorgehoben, um die Vorteile der Religion herunterzuspielen.

Zwischen Psychotherapeuten und Klerus besteht weiterhin ein Antagonismus, da sich die Interessengebiete in gewisser Weise überschneiden und oft dieselben «Personen/Kunden/Patienten(?)» teilen.

Zudem ist die Befürchtung einiger Psychotherapeuten bekannt, dass das Besprechen religiöser Themen einen Weg in unbekanntes Gebiet darstellen könnte. Sie laufen Gefahr, dem Patienten selbst Schaden zuzufügen. Psychotherapeuten können sich unwohl fühlen, wenn sie sich in ein soziales Versorgungsnetzwerk eingebunden fühlen, in dem die Rollen zwischen Ärzten und Religionsangehörigen nicht genau definiert sind.

Auch sonstige hemmende Faktoren in der Kommunikation zwischen Psychotherapeut und Patient gilt es bei der Auseinandersetzung mit der religiösen oder spirituellen Frage zu berücksichtigen. Faktoren, die das komplexe Verhältnis zwischen Medizin und Religion bestimmen. Das zu private Problem: der Therapeut, der seine eigene Religion durchsetzen will oder zu wenige Kenntnisse über das Verhältnis zwischen Religion und Gesundheit hat, und schliesslich Patienten, die nicht darüber sprechen wollen (Neeleman \& King, 1993). Ein gemeinsamer Faktor in allen diesen Elementen ist die fehlende Kompetenz in Sachen Religion und Spiritualität sowie der Mangel an verfügbaren Werkzeugen.

Auf globaler Ebene, bei Personen mit Störungen der psychischen Sphäre, ist es entscheidend, dass ihr Leiden sowohl in wissenschaftlicher als auch in spiritueller Hinsicht erfasst wird, um ihre Therapieadhärenz zu maximieren und die therapeutische Beziehung zu stärken.

\section{Beziehung zwischen Religion, Medizin und Psychiatrie}

Der Prozess der psychiatrischen Einschätzung muss auf die Ethnie des Subjekts, den Geburtsort, das Geschlecht, die soziale Klasse, die sexuelle Orientierung sowie auf seine religiösen und spirituellen Überzeugungen ausgerichtet sein (American Psychiatric Association, 1995).

Die Forschung weist uns darauf hin, dass Religion und/oder Spiritualität Menschen mit somatischen Störungen hilfreich sein können. So sind beispielsweise Angehörige religiöser Gruppen besser vor Herzbeschwerden geschützt (Goldbourt et al., 1993). Dies kann auf die Korrelation zwischen religiösen Überzeugungen und kardiovaskulären Risikofaktoren wie Bluthochdruck, Rauchen, schlechte Essgewohnheiten zurückzuführen sein, die in ihrer Stresswirkung durch religiöses Verhalten reduziert werden.

Religion könnte auch die Krebsinzidenz beeinflussen, insbesondere durch korrekte Essgewohnheiten und gesunde Praktiken, die in einigen religiösen Gruppen gepflegt werden (Enstrom, 1989). Die Entwicklung und die Befunde von Tumoren könnten auch dank religiösem Engagement durch verbessertes gesundes Verhalten günstig beeinflusst werden. Aber auch der Einsatz von religiösen Copingstrategien kann helfen, Hoffnung zu wecken und Angst abzubauen.

Aus vorwiegend historischen Gründen war in der Psychiatrie die allgemeine Einstellung zur Religion stets ambivalent. Religiöse Überzeugungen, Praktiken und direkte Erfahrungen wurden von den Fachleuten der psychischen Gesundheit - zumindest früher - oft als Formen von Neurose eingestuft. Die Religion bietet eine alternative Sichtweise der psychiatrischen Störungen, die mit Psychiatern und Psychotherapeuten in Konflikt geraten kann. 
Es hat sich gezeigt, dass religiöse Bewältigungsstrategie die Ergebnisse der Trauerverarbeitung und schwerer depressiver Störungen beeinflussen kann (Mohr \& Huguelet, 2004). Untersuchungen, die in der Schweiz von einigen Autoren und auch im Ausland durchgeführt wurden, haben den positiven Nutzen in Bezug auf Coping dokumentiert, der Religion und/oder Spiritualität für psychotische Patienten haben kann (Mohr \& Huguelet, 2004; Mohr et al., 2006; Yangarber-Hicks, 2004).

Psychotherapeuten sollten sich mit diesem Thema befassen und mit ihren Patienten immer über ihre religiösen Praktiken sprechen. Es ist wichtig, nicht nur zu wissen, ob sie gläubig sind, sondern auch die Intensität ihrer religiösen Gefühle und ihre spirituelle und religiöse Praxis zu kennen. Colucci und Martin (Colucci \& Martin, 2008) haben das wichtigste Modell der empirischen Forschung zwischen Religion und Selbstmord beschrieben.

Die Autoren kommen zum Schluss, dass religiöse Faktoren im Allgemeinen mit einer niedrigeren Selbstmordgedanken, einer negativeren Einstellung zum Selbstmord und niedrigeren Selbstmordversuchsraten verbunden sind. Kurz gesagt, Religion scheint einen besseren Schutz vor selbstmörderischen Gedanken und Verhaltensweisen zu bieten, dank einer besseren sozialen Integration (z. B. Zugehörigkeit zu religiösen Gruppen), dem Inhalt religiöser Überzeugungen oder der Einhaltung sozialer Normen, die selbstmörderische Überlegungen nicht unterstützen (Colucci \& Martin, 2008).

\section{Neurobiologische Grundlagen von Religion und Spiritualität}

Die Ergebnisse der Meta-Analyse deuten darauf hin, dass ein hohes Mass an Spiritualität mit der Aktivierung des serotonergen und dopaminergen Systems verbunden ist. Der Frontallappen, der präfrontale Kortex und die Verbindungen mit dem Thalamus, der hintere und obere Parietallappen und das Limbische System sind die am stärksten betroffenen Regionen (Newberg et al., 2001, 2003; Newberg \& Iversen, 2003; Muramoto, 2004).

Einige Studien über die Aktivierung der serotonergen und dopaminergen Wege deuten darauf hin, dass höhere Aktivitätsniveaus in den beiden Systemen nicht nur bei Personen mit einem hohen Sinn für Spiritualität, sondern auch bei religiösen Praktiken wie Meditation und Kontemplation vorhanden sind (Cloninger et al., 1993, 1998).

Hoffnung, Optimismus, Liebe und Zufriedenheit stimulieren die Produktion von Endorphinen, indem sie eine Aktivierung des Genuss-Kreislaufs induzieren und so Störungen im psychiatrischen Bereich verhindern. Durch die Aktivierung des limbischen Systems und der Hypothalamus-Hypophysen-NebennierenrindenAchse stärken sie das Immunsystem und reduzieren den Wechsel von Stresshormonen, wodurch unter anderem Herzfrequenz, Blutdruck, Angst und Depression vermindert werden.

Spiritualität und Religion können durch die oben beschriebenen neurobiologischen Mechanismen eine
Pufferwirkung auf die Stressoren des Lebens haben. Als Quelle der inneren Gelassenheit sorgen sie für ein Gefühl der Zufriedenheit und für Schutz vor pathologischen Auswirkungen.

\section{Klinische Fälle}

Der erste vorgelegte klinische Fall betrifft eine 66-jährige katholische Frau. Ihre Religiosität beruht auf dem Modell rigoroser, praktizierender katholischen Eltern. Die ganze Familie ging jeden Sonntag zur Messe und nahm aktiv an den Funktionen und Aktivitäten der Pfarrgemeinde teil. Sie ist Primarschullehrerin von Beruf, verheiratet und Mutter von drei adoptierten Kindern. Sie war zur Therapie wegen einem Problem in ihrer Beziehung zu ihrem Sohn gekommen, der damals Marihuana konsumierte. Sie zeigte ausserdem ein depressives Bild.

Es gab eine Diagnose von Brustkrebs im Jahr 2005, Blasen- und Eierstockkrebs im Jahr 2008. Jedes Mal, unter Befolgung der spezifischen onkologischen Behandlungen und gleichzeitiger Adhärenz an Gebets- und Kontemplationspraxis hat sie die Krebsleiden überwunden. «Wenn ich zwischen dem Krebs und dem Kind wählen muss, dann wähle ich den Krebs ... der Krebs ist ein Spaziergang für mich ...». So drückte sie ihre Ängste gegenüber dem eigenen Sohn aus. Sie zeigte oft sehr tiefe Angstkrisen, mit Unruhe, Spannung, Schlaflosigkeit, in einer Persönlichkeit mit invasiven und hyper-kontrollierenden Zügen.

Im Verlauf der individuellen systemischen Psychotherapie haben wir mit Unterstützung einer spezifischen Pharmakotherapie den religiösen Bereich einbezogen, der in ihrer persönlichen Geschichte und ihrem Alltag besonders ausgeprägt ist. Die Teilnahme an Morgengebeten und der Abendmesse wurde verstärkt.

Als psychosoziale Faktoren der Religions-Adhärenz zum Schutz vor der Krankheit haben sich herausgestellt: Zugehörigkeit zur Gruppe, Sozialisation, Kontemplation im Gebet mit einer daraus resultierenden Verringerung der Symptome und der Fähigkeit, Bereiche mit subjektivem Trost zu identifizieren. Wir konnten auch eine Verringerung der Wutzustände und der Impulsivität sowie der obsessiven Grübeleien verzeichnen (insbesondere über den Sohn).

Der zweite klinische Fall hat die Geschichte eines 28-jährigen Mannes zum Inhalt. «Die Schnur hatte sich wie eine Schlinge an meinem Hals gelegt ... mein Vater, ein Mann mit schlechtem Charakter, egoistisch, war gewalttätig gegen meine Mutter und gegen mich ...» Wegen der erlittenen Misshandlungen beantragte und erreichte er als Erwachsener die Änderung seines Nachnamens, der ihn mit seinem Vater identifizierte. Seit der Trennung seiner Eltern lebt er ausschliesslich bei seiner Mutter. Er hat einen älteren und einen jüngeren Bruder; die Beziehung zu ihnen ist dysfunktional.

Er wurde in der Mittelstufe gemobbt und wegen Übergewicht ausgelacht. Er hat ein Philosophiestudium an der USI (Universität der italienischen Schweiz) in Lugano abgeschlossen. 
In seiner persönlichen Geschichte taucht die relationale Isolation als spezifisches evolutionäres und aktuelles Merkmal auf. Auf der diagnostischen Seite befinden wir uns im Bereich einer narzisstischen Persönlichkeitsstörung mit ausgeprägt obsessiven Merkmalen, zusammen mit dem depressiven Bereich.

Nach einer schweren relationalen Frustration, als ihm zu Unrecht vorgeworfen wurde, Gegenstände von einer Gruppe von Freunden gestohlen zu haben, die er als Ersatz für seine auseinandergebrochene Familie gewählt hatte, wurde er aus der Gruppe verstossen.

Danach tauchte er in einen Zustand der Wut mit einem starken reaktiven Drang, dem Islam beizutreten. Er war sich bisher seiner katholischen Religion bewusst, hatte jedoch keine spirituelle oder soziale Beziehung zu religiösen Praktiken katholischer oder christlicher Natur.

Wenige Wochen nach seiner Bekehrung zum Islam präsentierte er sich kahlgeschoren und mit langem Bart. «Ich bin Muslim geworden ... Ich will den Koran auswendig lernen, auf Arabisch ...» Nach ein paar Wochen erklärte er: «Ich habe alle Medikamente verbrannt, ich will nicht mehr narkotisiert werden ...» Er wurde vom fehlgeleiteten Islam indoktriniert, in der fundamentalistischen, aggressiven Form, mit «extremistischen» Strömungen gegen die westliche Zivilisation und den amerikanischen Imperialismus.

Nach einigen Monaten wurde er in die Kantonale Psychiatrische Klinik Mendrisio eingewiesen, weil er sehr aggressiv - auch physisch - gegen seine Mutter geworden war. Die Mutter duldete nicht, dass er ihr die islamischfundamentalistische Religion aufzwingen wollte.

Nach dieser Krise wandte sie sich vom extremistischen und aggressiven Islamismus ab. Ein Jahr später kam es zu einer erneuten Annäherung an den Islam.

«Als ich letzten Sommer dem Islam beitrat, war ich von dieser Religion fasziniert ... jetzt wurde mir klar, dass ich mit ihren Grundwahrheiten nicht einverstanden bin, auch wenn sie die einzige monotheistische ist, an die ich glaube ... der Gedanke an Religion ist aktiv zurückgekehrt, im Gehirn, im Geist ... die Wahrheiten des Islamismus sind offenbar ... Ich vermisse das Gebet ... Ich falle aus jedem Anlass in eine Krise und weil ich mich vom Islam entfernt habe ... Ich habe ein Gefühl von Schuld und Schmerz und grosser Orientierungslosigkeit ... Ich habe keine Bezugspunkte ... Der Islam wird zu meinem Bezugspunkt ...»

Er besucht regelmässig die Moschee. Am Samstagabend trifft er sich mit einer Gruppe junger Menschen im Alter zwischen 18 und 30 Jahren, um gemeinsam den Islam zu studieren, zu beten und zu speisen. Am Sonntag geht er mit dem Imam in die Moschee, um den Koran zu lernen und zu rezitieren, er isst mit anderen Jungen zu Mittag. «Diesmal bin ich nicht zum Islam zurückgekehrt, nicht mit der politischen Idee des Heiligen Krieges, sondern mit der Idee der Religion.»

Die Analyse dieses Falles zeigt, welches die erleichternden Auswirkungen der Religion auf die Pathologie sein können, wenn sie eine tiefe existentielle Leere ersetzt, insbesondere als Gegenreaktion auf eine tiefe Frustration und Demütigung.

Die erste Annäherung an den Islam war integralistischer Natur, auf der Suche nach einer definierten, ja sogar gewalttätigen Rolle beim Neuaufbau einer zerbrochenen Persönlichkeit. Der erzwungene Klinikaufenthalt wirkte wie ein gegenpsychisches Trauma und brachte ihn zurück zu einer Wahl, die seinem Ich näher lag. Seitdem wird die Religion nicht mehr als geschlossene, integralistische «Sekte» erlebt, sondern als offener Raum für das psychische und soziale Selbst. Sie ist jetzt ein Schutz vor Pathologie geworden und bewahrt ihn vor möglichen zukünftigen psychischen Störungen.

Er stellt die folgenden Vorteile fest: Gelassenheit, Zugehörigkeit, Sozialisation, signifikante Reduzierung der Aggressionszustände, bessere Beziehung zu den Bindungspersonen.

Der dritte klinische Fall betrifft einen Mann, geboren im Jahr 1964 in Indien, in Punjab. Sein Vater starb, als er acht Jahre alt war. Die Mutter lebt noch. Er wurde als vierter Sohn in einer Familie mit zwei Brüdern und einer Schwester geboren. Nach dem Gymnasium wurde er für sechseinhalb Jahre in die Armee eingezogen. Er nahm aktiv am Krieg in Sri Lanka teil.

Aufgrund der Gefahr, aus politischen Gründen in Indien inhaftiert zu werden, entschied er sich, in die Schweiz auszuwandern. Er kam 1993 als politischer Flüchtling an. Nach einigen Jahren erhielt er eine Aufenthaltserlaubnis. In Indien war er verheiratet. Er kam allein in die Schweiz und liess seine Familie - die Frau und die beiden Kinder - in Indien zurück. Im Jahr 2004 wurde die Ehe geschieden.

In diagnostischer Hinsicht befinden wir uns im depressiven Bereich mit traumatischen Elementen, verbunden mit schweren Erlebnissen, ausgelöst durch verheerende Kriegserfahrungen. Er gehört dem hinduistischen religiösen Bereich an. Jeden Morgen praktiziert er etwa eine Stunde lang hinduistische Anbetung (Puja) vor einem kleinen Tempel zu Hause: «Das hat mir immer geholfen bei Angst und Depression.»

Das Ritual findet immer vor diesem kleinen Tempel statt, mit Düften und Kerzen. Er hat ein Buch, aus dem er liest.

«... man spricht vor Gott, er hört zu, ich fühle in mir Treue und Vertrauen, dass er mich ansieht und mir hilft. Seitdem ich mich krank fühle, tue ich das ... früher tat ich es nicht ... ich bete, damit Gott mich nicht verlässt, weil ich Angst habe, dass mir etwas zustossen könnte ... in Indien gibt es viele Orte, an denen frisches Wasser und Milch hervorquellen und die Menschen trinken und es geht ihnen besser ... Gott existiert also ...»

Die schützenden Faktoren gegen die Entwicklung einer ernsteren Psychopathologie waren: Abbau von Angst und Einsamkeitsgefühl, Abbau von negativen depressiven Gedanken, starkes Zugehörigkeitsgefühl, Aufhebung von selbstunterdrückenden Gedanken. 
Der vierte klinische Fall veranschaulicht die Geschichte einer Frau im Jahr 1970. Ihre Familie stammt aus den Abruzzen, sie ist in der Schweiz geboren und aufgewachsen. Der Vater wird symbolisch als «Padre Padrone» (MeisterVater) definiert. Die Mutter ist immer eine unterwürfige Frau gewesen. Sie hat einen Bruder und war erstmals 1992 verheiratet und 1995 geschieden. Aus der Ehe stammt eine Tochter, die an einem schweren Morbus Crohn mit Analfisteln leidet. Ein zweites Mal heiratet sie im Jahr 2013.

Diagnostisch befinden wir uns im Bereich einer somatoformen vegetativen Störung mit einem Zustand der Grundangst und Panikkrisen. Die gezeigten Symptome lagen im Bereich der Unfähigkeit, Feststoffe zu schlucken. Sie hatte auch starke Sorgen wegen ihrer Tochter, die sich seit vielen Jahren spezifischen Behandlungen ohne wirklichen Nutzen unterzieht. Als Christin fand sie besonders starke Unterstützung in der evangelischen Glaubensgemeinde. Seit 2009 besucht sie die Evangelische Kirche.

Die offensichtlichen Vorteile der Zugehörigkeit zur evangelischen Gemeinde waren: Verbesserung der Angstzustände, signifikante Reduktion des Symptoms mit der erneuten Fähigkeit, feste Nahrung zu sich zu nehmen. Sie hat ihr Selbstvertrauen gestärkt. Es ist ihr gelungen, dem männlichen Geschlecht Vertrauen zu geben und hat sich zugestanden, sich zu verlieben und zu heiraten: eine signifikante Reduzierung der thymischen Krisen. Psychotherapeutisch wurden eine individuelle kognitive Verhaltenstherapie sowie eine individuelle systemische Beziehungstherapie festgelegt.

Sobald das Therapiesystem für religiöse Überzeugungen empfänglich wurde und sie in den Behandlungsprozess integrierte, traten eine deutliche Beschleunigung der klinischen Verbesserung und des Zugehörigkeitsgefühls ein.

Der fünfte klinische Fall steht im Zusammenhang mit der komplexen Geschichte eines Mannes Jahrgang 1982, geboren und aufgewachsen in der Schweiz, mit nicht praktizierenden katholischen Eltern. Sein Vater ist Arzt mit einer bipolaren Erkrankung, die ihn in den letzten Jahren an der Ausübung seines Berufes gehindert hat. Der Vater wurde mehrmals in eine psychiatrische Klinik eingeliefert, ebenso wie seine Frau.

Die Mutter, von Beruf Krankenschwester, ist depressiv und ängstlich. Ein erster Bruder mit depressivem Syndrom befand sich auch einmal in psychiatrischer Behandlung. Die jüngere Schwester leidet an einer schweren Essstörung. Sie wurde in spezifische Kliniken eingewiesen. Er absolvierte die Matura, nachdem er das letzte Jahr viermal wiederholt hatte.

Diagnostisch gesehen befinden wir uns im Bereich der sozialen Angst mit Panikattacken und einem depressiven Zustand. Die Symptomatik, unter der er am meisten leidet, ist jedoch die des Schmerzbereichs.

«Meine Augen tun den ganzen Tag weh, nicht ein akuter, aber anhaltender Schmerz, schwere Augen. Ich muss mir den ganzen Tag die Nase schneuzen, meine verstopfte Nase ist störend ... Mein Hals schmerzt, seit einigen Monaten bereitet er mir Probleme bei der Arbeit ... mein Rücken tut den ganzen Tag lang weh, schon beim Aufwachen, er macht mir Probleme bei der Arbeit ... aber ich kann arbeiten, da ich stark bin. Meine Arme schmerzen, wenn ich vom Handgelenk an den Arm berühre, sogar meine Hände tun mir weh ... Ich habe Mühe, mich zu bewegen ... mein Brustkorb schmerzt im oberen Bereich und der Schmerz gleitet dann seitlich bis zu den Hüften. Der Oberschenkel schmerzt, der vordere Bereich des Oberschenkels, der Schmerz ist überall ... er breitet sich dann auf das Gesäss, die Genitalien, die Zunge, die Kopfhaut, die Finger und die Fusssohlen, den Hals, die Bronchien, die Lunge, das Pleura und das Herz aus ...»

Seit einigen Jahren interessiert er sich für die buddhistische Religion.

«Ich bin Buddhist geworden, weil ich in einer spirituellen Dimension leben wollte und etwas brauchte, das mir in schwierigen Zeiten Halt geben würde ... zuerst hatte ich Schwierigkeiten, es war mir klar, dass sich der buddhistische Ansatz stark von unserer schnellen und turbulenten Welt unterscheidet ... Der Buddhismus führt uns dazu, anzuhalten ... zuerst fällt es sehr schwer, weil ich Angst und Befürchtungen hatte, die mich blockierten, ... jetzt weiss ich, dass ich Angst mit positiven Emotionen bekämpfen kann ...»

Die Vorteile, die er unterstreicht, beziehen sich auf die Meditationspraxis am Morgen und Abend. «Sie können mich dazu bringen, zu kämpfen und die Schmerzen zu ertragen ...» Er spürt, dass er seine Schmerztoleranz gestärkt hat. Die Schmerzschwelle stieg und die Wahrnehmung des Schmerzes sank.

Im Rahmen der Psychotherapie erlaubte die Sensibilität für den spirituellen Bereich und die therapeutische Neugierde (Cloninger et al., 1993), die buddhistische Dimension in den Therapieverlauf einzubeziehen. Der Therapeut sollte eine Religionszugehörigkeit nie als definitiv erworben betrachten.

In der Tat, in diesem klinischen Fall zeigt sich, dass die erste Religion des Patienten die christlich-katholische Religion war. Nur das erweiterte Interesse und die spezifischen Fragen zur Spiritualität haben es uns ermöglicht, nachzuvollziehen und zu verstehen, woher die klinische Verbesserung kommt.

Es lag nicht nur an den Medikamenten, an der Kombination verschiedener psychotherapeutischer Ansätze, die im Laufe der Jahre angewandt wurden, von der systemischen Familientherapie über die kognitive Verhaltenstherapie, die Achtsamkeit, bis zum autogenen Training, sondern auch der Anreiz, den Weg des fortschreitenden Buddhismus einzuschlagen.

Der letzte dargestellte klinische Fall betrifft die Geschichte einer 71-jährigen Frau, die in Kampanien, einer Region in Süditalien, geboren und aufgewachsen ist. In den 1970er Jahren wanderte sie in die deutschsprachige Schweiz aus.

Ein erstes Mal war sie mit einem gewalttätigen Mann verheiratet, dann geschieden und nach ein paar Jahren 
erneut verheiratet. Später entdeckte sie, dass ihr zweiter Mann, der eine starke narzisstische Persönlichkeit hat, gleichzeitig ein paralleles Leben mit vielen anderen Frauen geführt hatte. Ihr gegenüber war der Ehemann unterdrückend, er verfolgte sie und schränkte ihre Freiheiten ein. Vor einigen Jahren gelang es ihr, sich von ihm zu trennen und beschloss, die deutschsprachige Schweiz zu verlassen und ins Tessin zu ziehen.

Aus diagnostischer Sicht kommt der depressive Bereich in einer persönlichen Geschichte zum Vorschein, die durch gestörte Beziehungen gekennzeichnet ist. Diese Beziehungen haben ihre persönliche Entwicklung stark behindert. Sie war schon immer eine christliche Katholikin, stark gläubig. In den komplexesten Phasen ihres Lebens hat sie ihre Zugehörigkeit zu Gebetsgruppen immer wieder verstärkt. Dabei erlebte sie eine starke Mystik, die manchmal Ausmasse annahm, die von psychiatrischer Seite als Eintreten eines religiösen Deliriums interpretiert werden könnten.

Indem sie die religiösen Praktiken, die sie auf ihrem Lebensweg befolgte, integriert hat und da es immer schützende Praktiken waren, auch wenn sie zu Deliriumartigen Momenten führen konnten, hat der Therapeut sie zu einem aktiven Teil des Settings gemacht. Damit stärkte er das Vertrauen in die Therapie und ermächtigte die Frau, in eine sichere, nicht aggressive oder disqualifizierende Umgebung einzutreten.

Die offenbarten Vorteile liegen in der Überwindung der schlimmsten Krisen durch Gebet und Kontemplation. Durch Gebet und Kontemplation erreicht sie eine deutliche Reduzierung ihrer von Angst- und Unruhezustände und kann sogar Phänomene der Wahrnehmungsstörung (Schatten und Stimmen) überwinden, die sie normalerweise in Momenten starker Krise erlebt. Es war sehr heilsam, die Phänomene der Wahrnehmungsstörung im therapeutischen Umfeld anzugehen. Die Öffnung des Settings für diese Aspekte hat es dem Geist ermöglicht, dem Risiko abweichender Wahnvorstellungen zu entkommen.

Die unvoreingenommene und nicht stigmatisierende Rezeption hat das Risiko einer psychotischen Drift aufgelöst und religiöse Praktiken wurden auf eine Besonderheit der persönlichen Geschichte zurückgeführt und haben den Charakter einer Selbstbehandlung angenommen.

\section{Schlussfolgerungen}

Aus den oben beschriebenen klinischen Fälle geht klar hervor, wie in der buddhistischen und hinduistischen Praxis die Meditation, die Beziehung zur Göttlichkeit, die starke Konnotation der Spiritualität - auch ohne die aktive und praktizierende religiöse Komponente zu einer Stärkung des eigenen Ichs führen kann. In der christlichen Religion hat die aktive Praxis eine Stärkung von Gruppensinn, Sozialisation und Integration offenbart, auch durch gemeinsames Beten und Treffen. Weitere wichtige Punkte waren die bessere Adhärenz an soziale
Normen, ein Zustand der gemeinsamen Kontemplation und die Schaffung eines starken Unterstützungsnetzes.

Spiritualität ihrerseits kann eine schützende Wirkung auf die Stressoren des Lebens haben, indem sie ein Gefühl des inneren Friedens aufbaut und stärkt, das in der Lage ist, sie zu kontrollieren. Bei der Analyse von klinischen Fällen und der Literatur wird es für jeden Therapeuten wichtig, in seiner Krankengeschichte die Merkmale von Religiosität, Spiritualität, aber auch von Atheismus zu sammeln.

Es wird von Bedeutung sein, die Fähigkeit zu entwickeln, mit Patienten über Spiritualität, Religion und Glauben zu sprechen und ein Gefühl von Empathie und Respekt zu vermitteln. Die Entwicklung der Fähigkeit zur Unterstützung und Stärkung der spirituellen und religiösen Bewältigung (Coping), wie Kontemplation, Meditation, Gebete, Teilnahme an Treffen mit Zugehörigkeitsgruppen, ist stark therapeutisch.

Die therapeutische Wirkung verläuft über die Stärkung des therapeutischen Bündnisses. Es regt die Verbesserung des Wohlbefindens an, mit der Folge, dass depressive und Angstzustände, der Konsum von Alkohol und Drogen sowie die Selbstmordrate zurückgehen. Das Vorhandensein eines strukturierten positiven Zusammenhangs zwischen Religion und Wohlbefinden kann ein Gefühl der inneren Ruhe und eine grössere Stabilität wiederherstellen. Durch die Stärkung der Religionszugehörigkeit oder der spirituellen/religiösen Praktiken kann das Subjekt andere psychosoziale Verbindungen entwickeln, die helfen können, den Behandlungs- und Verbesserungsprozess zu stärken.

Die therapeutische Beziehung kann immer persönlicher werden, indem die von Rogers formulierten unspezifischen therapeutischen Faktoren verstärkt werden: Unverfälschtheit, Empathie und ein bedingungslos positiver Blick (Rogers, 1957).

In einem Zeitalter, das von einem starken Gefühl der Ernüchterung, Akzentuierung der Einsamkeit und einem Gefühl der verlorenen Hoffnung geprägt ist, stellt dies eine Stärkung der klinischen Beziehung und der therapeutischen Sensibilität und schliesslich der Behandlung des Subjekts dar.

\section{Literatur}

American Psychiatric Association. (2000). Diagnostic and statistical manual of mental disorders (4th ed., text rev.). Washington, D. C.

Cecchin, G., Lane, G. \& Ray, W.A. (1997). Verità e Pregiudizi. Un approccio sistemico alla psicoterapia. Mailand: Raffaello Cortina.

Cloninger, C.R., Bayon, C. \& Svrakic, D. M. (1998). Measurement of temperament and character in mood disorders: a model of fundamental states as personality types. Journal of Affective Disorders, $51(1), 21-32$.

Cloninger, C.R., Svrakic, D. M. \& Przybeck, T.R. (1993). A Psychobiological Model of Temperament and Character. Archives of General Psychiatry, 50(12), 975-990.

Colucci, E. \& Martin, G. (2008). Religion and Spirituality Along the Suicidal Path. Suicide and Life-Threatening Behavior, 38(2), 229-244.

Enstrom, J.E. (1989). Health Practices and Cancer Mortality among Active California Mormons. Journal of the National Cancer Institute, 81, 1807-1814. 
Gay, P. (1987). A Godless Jew: Freud, Atheism, and the Making of Psychoanalysis. New Haven: Yale University Press.

Goldbourt, U., Yaari, S. \& Medalie, J.H. (1993). Factors Predictive of Long-Term Coronary Heart Disease Mortality among 10,059 Male Israeli Civil Servants and Municipal Employees. A 23-Year Mortality Follow-up in the Israeli Ischemic Heart Disease Study. Cardiology, 82, 100-121.

Koenig, H. G., McCullough, M.E. \& Larson, D. B. (2001). Handbook of Religion and Health. Oxford: University Press.

Koenig, L. B., McGue, M., Krueger, R. F. \& Bouchard Jr., T. J. (2005). Genetic and Environmental Influences on Religiousness: Findings for Retrospective and Current Religiousness Ratings. Journal of Personality, 73(2),471-488.

Larson, D. B., Swyers, J. P. \& McCullough, M.E. (1997). Scientific Research on Spirituality and Health: A Consensus Report. Rockville/ MD: National Institute for Health Research.

Mattia, M., Barbetta, P. \& Pakman, M. (2015). Abstract 4 congresso mondiale WACP.

Mohr, S., Brandt, P. Y., Gillieron, C., Borras, L. \& Huguelet, P. (2006). Toward an Integration of Spirituality and Religiousness Into the Psychosocial Dimension of Schizophrenia. American Journal of Psychiatry, 163, 1952-1959.

Mohr, S. \& Huguelet, P. (2004). The Relationship between Schizophrenia and Religion and Its Implications for Care. Swiss Medical Weekly, 134, 369-376.

Muramoto, O. (2004). The role of the medial prefrontal cortex in human religious activity. Medical Hypotheses, 62(4), 479-485.

Neeleman, J. \& King, M.B. (1993). Psychiatrists' religious attitudes in relation to their clinical practice: A survey of 231 psychiatrists. Acta Psychiatrica Scandinavica, 88, 420-424.

Newberg, A., Alavi, A., Baime, M., Pourdehnad, M., Santanna, J. \& D'Aquili, E. (2001). The Measurement of Regional Cerebral Blood Flow during the Complex Cognitive Task of Meditation: A Preliminary SPECT Study. Psychiatry Research, 106(2), 113-122.

Newberg, A. \& Iversen, J. (2003). The Neural Basis of the Complex Mental Task of Meditation: Neurotransmitter and Neurochemical Considerations. Medical Hypotheses, 61(2), 282-291.

Newberg, A., Pourdehnad, M., Alavi, A. \& D’Aquili, E. G. (2003). Cerebral Blood Flow During Meditative Prayer: Preliminary Findings and Methodological Issues. Perceptual and Motor Skills, 97(2), 625-630.

Rogers, C.R. (1957). The Necessary and Sufficient Conditions of Therapeutic Personality Change. Journal of Consulting Psychology, 21(2), 95-103.

Yangarber-Hicks, N. (2004). Religious Coping Styles and Recovery from Serious Mental Illness. Journal of Psychology and Theology, $32,305-317$
Spirituality, migration and psychiatry. A heated argument for Psychotherapy?

Abstract: Although medicine is practiced in a secular setting, religious and spiritual issues have an impact on patients' perspectives regarding their health and the management of disorders that may afflict them. This is especially true in psychiatry, because spiritual and religious beliefs are prevalent among those with emotional or mental illness. Clinicians are rarely aware of the importance of religion and understand little of its value as a positive force for coping with the many difficulties that patients and their families must face. In this paper I want to present some clinical cases to discuss the relationship between religion, spirituality and psychiatric disorder. Particularly the author will underline the pathofacilitator or the pathoprotective effect of Christian's, Muslim's, Buddhism's, Hinduism's and Evangelical's religion, through the analysis of specific clinical cases. The clinical cases showed will be focused on: psychosis, anxiety disorders, affective disorders, chronic pain disorder and somatic symptom disorder (Mattia et al., 2015).

Key Words: Cultural Psychiatry, Spirituality, Religion, Beliefs, Coping, Prejudice, Psychotherapy

\section{Der Autor}

Michele Mattia, FMH Psychiatrie und Psychotherapie, Präsident der Vereinigung der italienischen Schweiz für Angst, Depression und Zwangsstörungen (Asi-Adoc), Dozent an der Universität Varese, Psychiatrie; Lehrtätigkeit am Mailänder Familientherapiezentrum.

\section{Kontakt}

Viale San Salvatore 7 6902 Lugano-Paradiso Tel +41916471417

E-Mail: studiomattia@michelemattia.ch www. michelemattia.ch 